\title{
Geminal wavefunctions with Jastrow correlation: a first application to atoms
}

\author{
Michele Casula and Sandro Sorella \\ International School for Advanced Studies (SISSA) Via Beirut 2-4, 34014 Trieste, Italy \\ and INFM Democritos National Simulation Center, Trieste, Italy
}

(Dated: October 30, 2018)

\begin{abstract}
We introduce a simple generalization of the well known geminal wavefunction already applied in Quantum Chemistry to atoms and small molecules. The main feature of the proposed wavefunction is the presence of the antisymmetric geminal part together with a Jastrow factor. Both the geminal and the Jastrow play a crucial role in determining the remarkable accuracy of the many-body state: the former permits the correct treatment of the nondynamic correlation effects, the latter allows the wavefunction to fulfill the cusp conditions and makes the geminal expansion rapidly converging to the lowest possible variational energies. This ansatz is expected to provide a substantial part of the correlation energy for general complex atomic and molecular systems. The antisymmetric geminal term can be written as a single determinant even in the polarized cases. In general, therefore, the computational effort to sample this correlated wavefunction is not very demanding, the scaling of the algorithm with the number of atoms being comparable with the simplest Hartree-Fock calculation.

We applied this Jastrow-geminal approach to atoms up to $Z=15$, always getting good variational energies, by particularly improving those with a strong multiconfigurational nature. Our wavefunction is very useful for Monte Carlo techniques, such as Fixed node. Indeed, the nodal surface obtained within this approach can be substantially improved through the geminal expansion.
\end{abstract}

PACS numbers: 31.10.+z, 02.70.Ss, 02.70.Uu, 31.15.Ar, 31.15.Pf, 31.25.Eb, 71.10.Li

\section{INTRODUCTION}

One of the main goals in electronic structure calculations is to deal with a wavefunction both accurate to predict the physical properties of a quantum system and simple enough to allow feasible computations of them. In particular, although multideterminantal CI like methods could be in principle very accurate, in practice the determinantal expansion becomes heavier and heavier from the computational point of view, as the number of determinants dramatically increases with the complexity of the electron system. On the other hand, a single determinantal wavefunction, kernel of methods like Hartree-Fock (HF) and Density Functional Theory (DFT), is sometimes not sufficient to describe strongly correlated systems, as for instance the transition metal compounds and the near degenerate shell structure of some atoms.

Since 50's, the intensive efforts to explain theoretically the superconductivity have been highlighting the role of pairing in the electronic structure. The BCS wavefunction belongs to an original ansatz in which the correlation is introduced through the product of pairing functions (in this context called Cooper pairs), already exploited in quantum chemistry by the pioneering work of Hurley et al. 1] to treat correlation effects in molecular properties. Their wavefunction was called antisymmetrized geminal power (AGP) that has been shown to be the particleconserving version of the BCS ansatz [2]. It includes the single determinantal wavefunction, i.e. the uncorrelated state, as a special case and introduces correlation effects in a straightforward way, through the expansion of the pairing function (geminal): therefore it was studied as a possible alternative to the other multideterminantal approaches. Although this ansatz seemed so appealing, it led to some expensive optimization procedures [3, 4] with numerical problems [5, 6] in particular when applied to large systems, and so it turned out to be non competitive with respect to $\mathrm{HF}$ and $\mathrm{CI}$.

We show in this paper that the use of Monte Carlo methods can overcome the previous difficulties in optimizing the AGP wavefunctions. Two of the most appealing features of these techniques are the possibility to tackle in a smart way the many-body interacting problem, having the freedom in the choice of the functional form of the wavefunction, and to implement very efficient projection algorithms, like diffusion Monte Carlo (DMC) and Green's function Monte Carlo (GFMC). The trial wavefunction used in these methods is obtained by multiplying an antisymmetric term (usually called Slater term) to a Jastrow factor, which correlates the electrons and takes into account the interelectron cusp conditions the true wavefunction must fulfill. The Slater term can be either HF or CI or AGP like. As already pointed out by Umrigar [7], the rate of convergence of CI expansion is increased by the Jastrow factor, just because it allows the wavefunction to have the correct cusps, otherwise present only asymptotically in the linear combination of determinants.

In this paper we study the AGP-Jastrow $(A G P+J)$ wavefunction, applied to the atomic problem. The wavefunction we consider is actually a Resonating Valence Bond (RVB) state, first investigated in 1973 by Anderson [8, 9] to study the high-temperature superconductivity and later applied by Bouchaud and Lhuillier 10] in Monte Carlo calculations of liquid ${ }^{3} \mathrm{He}$. It is remarkable that this kind of wavefunction can describe well the ground state of all the atoms studied here, in particular those light atoms affected by nondynamic correlation. Moreover, the Jastrow part is crucial not only in reducing 
the pairing expansion but also in inducing a significant improvement of the nodal surfaces of the wavefunction, once we optimize both the Jastrow and the determinantal part at the same time. The minimization of the total energy can be efficiently performed by using an optimization procedure based upon the Stochastic Reconfiguration (SR) method [1], which allows us to determine a large number of variational parameters, both for the Jastrow and the determinantal part. Due to the simplicity of the $A G P+J$ wavefunction and its capability to take into account resonating Slater configurations, this wavefunction is expected to be appropriate also for molecules and more complex systems.

The paper is organized as follows: in Sec. [II we define the wavefunction, in Sec. III we describe the energy minimization method and finally in Sec. [V and $\nabla$ we present detailed results and conclusions.

\section{FUNCTIONAL FORM OF THE WAVE FUNCTION}

The wavefunction we used in our QMC calculations is the antisymmetrized product of geminals $(A G P)$, multiplied by a simple symmetric Jastrow factor:

$$
\Psi\left(\mathbf{r}_{1}, \ldots, \mathbf{r}_{N}\right)=\Psi_{A G P}\left(\mathbf{r}_{1}, \ldots, \mathbf{r}_{N}\right) J\left(\mathbf{r}_{1}, \ldots, \mathbf{r}_{N}\right),
$$

where the origin of the reference frame is the nuclear position. The two parts of the wavefunction (1) will be described in detail below.

\section{A. The determinantal part}

Let $\Phi$ be the pairing function (geminal) which takes into account the correlation between two electrons with opposite spin. If the system is unpolarized and the state is a spin singlet, the $A G P$ wavefunction is

$\boldsymbol{\Psi}_{A G P}\left(\mathbf{r}_{1}, \ldots, \mathbf{r}_{N}\right)=\hat{A}\left[\Phi\left(\mathbf{r}_{1}^{\uparrow}, \mathbf{r}_{2}^{\downarrow}\right) \Phi\left(\mathbf{r}_{3}^{\uparrow}, \mathbf{r}_{4}^{\downarrow}\right) \cdots \Phi\left(\mathbf{r}_{N-1}^{\uparrow}, \mathbf{r}_{N}^{\downarrow}\right)\right]$,

where $\hat{A}$ is an operator that antisymmetrizes the product in the square brackets and the geminal is a singlet:

$$
\Phi\left(\mathbf{r}^{\uparrow}, \mathbf{r}^{\downarrow}\right)=\phi\left(\mathbf{r}^{\uparrow}, \mathbf{r}^{\downarrow}\right) \frac{1}{\sqrt{2}}(|\uparrow \downarrow\rangle-|\downarrow \uparrow\rangle),
$$

implying that $\phi\left(\mathbf{r}, \mathbf{r}^{\prime}\right)$ is symmetric under a permutation of its variables. Given this conditions, one can prove 14 that the spatial part of the $\boldsymbol{\Psi}_{A G P}$ can be written in a very compact form:

$$
\Psi_{A G P}\left(\mathbf{r}_{1}, \ldots, \mathbf{r}_{N}\right)=\operatorname{det}\left(A_{i j}\right),
$$

where $A_{i j}$ is a $\frac{N}{2} \times \frac{N}{2}$ matrix defined as:

$$
A_{i j}=\phi\left(\mathbf{r}_{i}^{\uparrow}, \mathbf{r}_{j}^{\downarrow}\right) .
$$

We are going to extend the definition of the geminal wavefunction to a polarized system, i.e. a system with a different number of electrons for each spin. This generalization of the geminal model was first proposed by Coleman [15] and called GAGP. Without loss of generality, one can assume that the number of up particles $\left(N^{\uparrow}\right)$ is greater than the down ones $\left(N^{\downarrow}\right)$. In order to write down the many-body wavefunction with the geminals, one needs to introduce $N^{\uparrow}-N^{\downarrow}$ single particle spin orbitals $\bar{\Phi}_{j}$ not associated with any geminal and holding unpaired electrons. Once again one recovers the compact notation (44) for the spatial part of $\Psi_{A G P}$ (see Appendix), but this time $A_{i j}$ is a $N^{\uparrow} \times N^{\uparrow}$ matrix defined in the following way:

$$
A_{i j}=\left\{\begin{aligned}
\phi\left(\mathbf{r}_{i}^{\uparrow}, \mathbf{r}_{j}^{\downarrow}\right) & \text { for } j=1, N^{\downarrow} \\
\bar{\phi}_{j}\left(\mathbf{r}_{i}^{\uparrow}\right) & \text { for } j=N^{\downarrow}+1, N^{\uparrow}
\end{aligned}\right.
$$

where the index $i$ ranges from 1 to $N^{\uparrow}$.

The pairing function can be expanded over a basis of single particle orbitals [16]:

$$
\phi\left(\mathbf{r}^{\uparrow}, \mathbf{r}^{\downarrow}\right)=\sum_{i=1}^{M} \lambda_{i} \psi_{i}\left(\mathbf{r}^{\uparrow}\right) \psi_{i}^{*}\left(\mathbf{r}^{\downarrow}\right),
$$

where $\psi_{i}$ are general real or complex normalized functions and $\lambda_{i}$ are variational parameters that may depend on the chosen spatial symmetry of the geminal. Hereafter, for simplicity, we do not assume that the orbitals are mutually orthogonal.

For the application to atoms, in order the wavefunction $\Psi$ has a definite angular momentum, it is convenient that the geminal is rotationally invariant around the nucleus. This requirement is achieved by taking the generic orbital $\psi_{i}$ to be an eigenfunction of the single particle angular momentum operators $l^{2}$ and $l_{z}$; hence, the orbital will be denoted by:

$$
\psi_{n l m}(r, \theta, \phi)=R_{n l}(r) Y_{l m}(\theta, \phi),
$$

where $Y_{l m}$ are spherical harmonics with standard notations and the radial part $R_{n l}$ depends on the principal quantum number $n$. In this way, the atomic geminal function takes the form:

$$
\phi\left(\mathbf{r}^{\uparrow}, \mathbf{r}^{\downarrow}\right)=\sum_{n l} \sum_{m=-l}^{l} \lambda_{n l}(-1)^{m} \psi_{n l m}\left(\mathbf{r}^{\uparrow}\right) \psi_{n l m}^{*}\left(\mathbf{r}^{\downarrow}\right) .
$$

In the polarized case, the remaining orbitals $\bar{\psi}_{j}$ may change the total angular momentum and spin quantum numbers with the same rules valid for Slater-type wavefunctions. Within our ansatz it is therefore possible to have definite total spin and angular momenta at least in all cases when the conventional Slater function does.

The minimal geminal expansion is for $M=N^{\downarrow}$, then the $A G P$ wavefunction is reduced to the HF one; instead, if $M$ is greater than $N^{\downarrow}$, one can prove that the $A G P$ is 
equivalent to a linear combination of many Slater determinants. Therefore, within this functional form, one is able to introduce the correlation in the determinantal part of the wavefunction just by adding some terms in the geminal expansion. For a two-electrons closed-shell system, like Helium atom, or an ensemble of such noninteracting systems, this wavefunction is exact. For the other atoms, we will show that the $A G P+J$ wavefunction can lead to very good variational energies, even in the cases where the HF approximation is rather poor, especially for light elements.

In order to optimize the radial part $R_{n l}$ of the the single particle orbitals, we expand these radial functions in a Slater basis, in close analogy with Roothaan-HartreeFock calculations [17], namely using functions of the type:

$$
r^{n-1} e^{-z_{k} r}
$$

with $n \geq 1$, taking in principle as many different $z_{k}$ 's as required for convergence.

In the Roothaan-Hartree-Fock the coefficients of the linear combinations are more involved, since the orthogonality among all single particle states is required. In the Monte Carlo approach we have found that it is much simpler and more efficient to deal with non-orthogonal orbitals, without spoiling the accuracy of the calculation. In fact, for light elements with $Z \leq 15$, studied here, it is possible to obtain almost converged results by using only two exponentials for each radial component (double zeta).

Hence, our single particle orbitals read in general

$$
R_{n l}(r)=C r^{n-1}\left(e^{-z_{1} r}+p e^{-z_{2} r}\right),
$$

where $p$ is another variational parameter and $C$ is the normalization factor for the radial part $R_{n l}$ :

$$
C=\frac{1}{\sqrt{(2 n) !}}\left(\left(2 z_{1}\right)^{-(2 n+1)}+2 p\left(z_{1}+z_{2}\right)^{-(2 n+1)}+p^{2}\left(2 z_{2}\right)^{-(2 n+1)}\right)^{-\frac{1}{2}}
$$

Actually $p$ is not free for all the orbitals: indeed, for a more accurate variational wavefunction, we impose the electron-nucleus cusp condition [18, 19], which is satisfied by the exact many body ground state and implies that each orbital must fulfill the following relation:

$$
\frac{\partial \hat{\psi}}{\partial r}=-Z \psi
$$

at $r=0$ (the hat denotes the spherical average). That condition is automatically obeyed by all but $1 s$ and $2 s$ orbitals of the type given in Eq[1] Instead, the parameter $p$ of $1 s$ orbital must be:

$$
p=\frac{z_{1}-Z}{Z-z_{2}}
$$

and for the $2 s$ state, in order to fulfill Eq. (13), we choose a functional form of the type:

$$
\psi_{2 s}(r)=e^{-z_{1} r}+(p+\alpha r) e^{-z_{2} r},
$$

where $\alpha$ is a further variational parameter and $p$ is given by

$$
p=\frac{z_{1}-\alpha-Z}{Z-z_{2}} .
$$

In our study, we found that the presence of the $\alpha$ term leads to a very marginal improvement of the variational wavefunction, therefore we set $\alpha=0$ and we kept the $1 s$ and $2 s$ orbitals to have the same functional form, in order to reduce at most the total number of parameters.

\section{B. The Jastrow factor}

The Jastrow factor in our wave function is very simple and has been widely used in previous Monte Carlo electronic structure calculations:

$$
J\left(\mathbf{r}_{1}, \ldots, \mathbf{r}_{N}\right)=\prod_{i<j} \exp \left(f\left(\mathbf{r}_{i}, \mathbf{r}_{j}\right)\right)
$$

where the product is over all pairs of electrons and for simplicity the function $f$ depends only on their relative distance $r_{i j}$ and their spins $\sigma_{i}$ and $\sigma_{j}$, namely:

$$
f_{\text {two body }}\left(r_{i j}\right)=\frac{a_{\sigma_{i} \sigma_{j}} r_{i j}}{1+b_{\sigma_{i} \sigma_{j}} r_{i j}} .
$$

The value of $a_{\sigma_{i} \sigma_{j}}$ is fixed by the cusp condition at the coalescence points of two electrons and $b_{\sigma_{i} \sigma_{j}}$ contains at most three free variational parameters, as $b_{\uparrow \downarrow}=b_{\downarrow \uparrow}$ is implied by the spatial symmetry of the Jastrow factor. The cusp conditions for parallel and antiparallel spin electrons are different and yield two different values of $a_{\sigma_{i} \sigma_{j}}$ :

$$
\begin{aligned}
& a=\frac{1}{2} \text { for } \sigma_{i}=\sigma_{j} \\
& a=\frac{1}{4} \text { for } \sigma_{i} \neq \sigma_{j} .
\end{aligned}
$$

As pointed out in Ref. 20, whenever $a_{\sigma_{i} \sigma_{j}}$ or $b_{\sigma_{i} \sigma_{j}}$ depend on the electron spins $\sigma_{i} \sigma_{j}$, the wavefunction will be spin contaminated, i.e. it will not be an eigenstate of 
the total spin operator $S^{2}$. We have chosen to preserve the correct spin symmetry of the total wavefunction, by keeping $a_{\sigma_{i} \sigma_{j}}=1 / 2$ and $b_{\sigma_{i} \sigma_{j}}=b$, hence fulfilling only the first condition (19a). Indeed, the cusp condition for electrons with parallel spins is much less important because their probability to get close is clearly small, due to the Pauli principle.

For the nitrogen atom, we checked the quality of this wavefunction with respect to a spin contaminated one with two variational parameters, $b_{\uparrow \uparrow}=b_{\downarrow \downarrow}=b_{1}$ and $b_{\uparrow \downarrow}=b_{2}$, both of them reported in Table III listed with the other atoms. In both the cases, we kept the geminal expansion to be minimal ( $H F+J$ like wavefunction). As reported in Table [I the improvement in energy obtained by contaminating the spin wavefunction is rather negligible, and disappears when the FN DMC simulation is carried out. This implies that it is possible to obtain almost optimal nodes, without spoiling the spin symmetry and by using only one variational parameter for the Jastrow factor.

In order to study the effectiveness of the Jastrow for a more accurate determination of the nodal surface, we have implemented a more involved Jastrow factor, including three-body correlation terms, and we have applied it to few atoms ( $B e$ and $M g$ ). Indeed the Jastrow term $f$ in Eq. 17 is a general function of the positions of two electrons and it has been parametrized similarly to the geminal function (17), but truncated up to the $l=1$ angular momentum:

$$
\begin{aligned}
f\left(\mathbf{r}_{i}, \mathbf{r}_{j}\right)= & f_{\text {two body }}\left(r_{i j}\right)+ \\
& \psi_{0}\left(\mathbf{r}_{i}\right) \psi_{0}\left(\mathbf{r}_{j}\right)+\bar{\psi}_{1}\left(\mathbf{r}_{i}\right) \cdot \bar{\psi}_{1}\left(\mathbf{r}_{j}\right) .
\end{aligned}
$$

The functional form for the s-wave $\left(\psi_{0}\right)$ and p-wave $\left(\bar{\psi}_{1}\right)$ components can be chosen among different types; the most widely used in the literature are the polynomial [21, 22] and the gaussian-polynomial mixed form [23]. In this work, we have selected the expansion over a gaussian basis, as reported in Table $\nabla$ This parametrization of the Jastrow factor, though being certainly less general compared with the best possible ones 24], includes the most significant part of the three-body correlation [25], which involves two electrons and the nuclei. Our purpose, in fact, is to check whether it is possible to lower significantly the energy of the $A G P+J$ wavefunction, whenever the Jastrow part of the wavefunction is systematically improved together with the determinantal part.

\section{METHOD}

\section{A. Minimization method}

We have performed the wavefunction optimization by using the stochastic minimization of the total energy based upon the Stochastic Reconfiguration (SR) technique, already exploited for lattice systems [11]. Let $\Psi_{T}\left(\alpha^{0}\right)$ be the wavefunction depending on an initial set of $p$ variational parameters $\left\{\alpha_{k}^{0}\right\}_{k=1, \ldots, p}$. Consider now a small variation of the parameters $\alpha_{k}=\alpha_{k}^{0}+\delta \alpha_{k}$. The corresponding wavefunction $\Psi_{T}(\alpha)$ is equal, within the validity of the linear expansion, to the following one:

$$
\Psi_{T}^{\prime}(\alpha)=\left(\Psi_{T}\left(\alpha^{0}\right)+\sum_{k=1}^{p} \delta \alpha_{k} \frac{\partial}{\partial \alpha_{k}} \Psi_{T}\left(\alpha^{0}\right)\right)
$$

Therefore, by introducing local operators defined on each configuration $x=\left\{\mathbf{r}_{1}, \ldots, \mathbf{r}_{N}\right\}$ as the logarithmic derivatives with respect to the variational parameters:

$$
O^{k}(x)=\frac{\partial}{\partial \alpha_{k}} \ln \Psi_{T}(x)
$$

and for convenience the identity operator $O^{0}=1$, we can write $\Psi_{T}^{\prime}$ in a more compact form:

$$
\left|\Psi_{T}^{\prime}(\alpha)\right\rangle=\sum_{k=0}^{p} \delta \alpha_{k} O^{k}\left|\Psi_{T}\right\rangle,
$$

where $\left|\Psi_{T}\right\rangle=\left|\Psi_{T}\left(\alpha_{0}\right)\right\rangle$ and $\delta \alpha_{0}=1$. In general, $\delta \alpha_{0} \neq$ 1 , in that case the variation of the parameters will be scaled

$$
\delta \alpha_{k} \rightarrow \frac{\delta \alpha_{k}}{\delta \alpha_{0}}
$$

and $\Psi_{T}^{\prime}$ will be proportional to $\Psi_{T}(\alpha)$ for small $\frac{\delta \alpha_{k}}{\delta \alpha_{0}}$.

Our purpose is to set up an iterative scheme to reach the minimum possible energy for the parameters $\alpha$, exploiting the linear approximation for $\Psi_{T}(\alpha)$, which will become more and more accurate close to the convergence, when the variation of the parameters is smaller and smaller. We follow the stochastic reconfiguration method and define

$$
\left|\Psi_{T}^{\prime}\right\rangle=P_{S R}(\Lambda-H)\left|\Psi_{T}\right\rangle
$$

where $\Lambda$ is a suitable large shift, allowing $\Psi_{T}^{\prime}$ to have a lower energy than $\Psi_{T}$ [11], and $P_{S R}$ is a projection operator over the $(p+1)$-dimensional subspace, spanned by the basis $\left\{O_{k}\left|\Psi_{T}\right\rangle\right\}_{k=0, \ldots, p}$, over which the function $\left|\Psi_{T}^{\prime}\right\rangle$ has been expanded (Eq. 23). Indeed, in order to determine the coefficients $\left\{\delta \alpha_{k}\right\}_{k=1, \ldots, p}$ corresponding to $\Psi_{T}^{\prime}$ defined in Eq25] one needs to solve the SR conditions:

$$
\left\langle\Psi_{T}\left|O^{k}(\Lambda-H)\right| \Psi_{T}\right\rangle=\left\langle\Psi_{T}\left|O^{k}\right| \Psi_{T}^{\prime}\right\rangle \text { for } k=0, \ldots, p
$$

that can be rewritten in a linear system:

$$
\sum_{l} \delta \alpha_{l} s^{l k}=f^{k}
$$

where $s^{l k}=\left\langle\Psi_{T}\left|O^{l} O^{k}\right| \Psi_{T}\right\rangle$ is the covariance matrix and $f^{k}=\left\langle\Psi_{T}\left|O^{k}(\Lambda-H)\right| \Psi_{T}\right\rangle$ is the known term; both $s^{l k}$ and $f^{k}$ are computed stochastically by a Monte Carlo integration. These linear equations (27) are very similar to the ones introduced by Filippi and Fahy [26] for the 
energy minimization of the Slater part. In our formulation, there is no difficulty to optimize the Jastrow and the Slater part of the wavefunction at the same time.

After the system (27) has been solved, we update the variational parameters

$$
\alpha_{k}=\alpha_{k}^{(0)}+\frac{\delta \alpha_{k}}{\delta \alpha_{0}} \text { for } k=1, \ldots, p
$$

and we obtain a new trial wavefunction $\Psi_{T}(\alpha)$. By repeating this iteration scheme several times, one approaches the convergence when $\frac{\delta \alpha_{k}}{\delta \alpha_{0}} \rightarrow 0$ for $k \neq 0$, and in this limit the SR conditions (26) implies the Euler equations of the minimum energy. Obviously, the solution of the linear system (27) is affected by statistical errors, yielding statistical fluctuations of the final variational parameters $\alpha_{k}$ even when convergence has been reached, namely when the $\left\{\alpha_{k}\right\}_{k=1, \ldots, p}$ fluctuate without drift around an average value. We perform several iterations in that regime; in this way, the variational parameters can be determined more accurately by averaging them over all these iterations and by evaluating also the corresponding statistical error bars.

It is worth noting that the solution of the linear system (27) depends on $\Lambda$ only through the $\delta \alpha_{0}$ variable. Therefore the constant $\Lambda$ indirectly controls the rate of change in the parameters at each step, i.e. the speed of the algorithm for convergence and the stability at equilibrium: a too small value will produce uncontrolled fluctuations for the variational parameters, a too large one will allow convergence in an exceedingly large number of iterations. The choice of $\Lambda$ can be controlled by evaluating the change of the wavefunction at each step as:

$$
\frac{\left|\Psi_{T}^{\prime}-\Psi_{T}\right|^{2}}{\left|\Psi_{T}\right|^{2}}=\sum_{k, k^{\prime}>0} \delta \alpha_{k} \delta \alpha_{k^{\prime}} s^{k k^{\prime}}
$$

By keeping this value small enough during the optimization procedure, one can easily obtain a steady and stable convergence.

Finally, we mention that the stochastic procedure is able in principle to perform a global optimization, as discussed in Ref. 11 for the SR and in Ref. 12 for the Stochastic Gradient Approximation (SGA), because the noise in the sampling can avoid the dynamics of the parameters to get stuck into local minima.

\section{B. Variational and Diffusion Monte Carlo}

We performed standard Variational (VMC) and Diffusion Monte Carlo (DMC), the latter within the so called Fixed Node (FN) approximation, which allows to obtain the lowest energy state with the same nodes of a trial wavefunction. As trial state for $\mathrm{FN}$, we have used the VMC wavefunction optimized with the SR method described in the previous section.

\section{RESULTS}

We have carried out Quantum Monte Carlo calculations for atoms from $L i$ to $P$, using the antisymmetrized geminal power times the Jastrow factor $(A G P+J)$ to describe the atomic electronic structure. We performed the optimization of both the geminal and the Jastrow part minimizing the energy with the method described in Sec III. For all the atoms, we considered the minimal geminal expansion, corresponding to the $H F$ single determinant, and the simplest Jastrow factor with a single parameter (18), reported in Table III To improve the antisymmetric part, we increased the number of orbitals in the geminal expansion, and for $\mathrm{Be}$ and $\mathrm{Mg}$ atoms we also systematically considered an improved Jastrow term, such as the three-body one described above (see Tables [V] and V]. As one can notice from the Tables, our wavefunction parametrization is very compact, even in the case of the mostly correlated states, since it contains always a relatively small number of parameters for each atom.

In order to judge the outcome of our calculations, we computed the correlation energies and in particular its fraction with respect to the exact ground state energy for the nonrelativistic infinite nuclear mass Hamiltonian, estimated in Ref. 13. The quality of the variational wavefunction can be seen by computing the expectation value of the energy by means of the VMC calculations. Furthermore, we carried out DMC simulations within the FN approximation, which allows to optimize the amplitude of the wavefunction inside each nodal volume, where its sign is given and fixed by the variational state. Therefore, the DMC energy depends on the quality of the nodal structure of the variational wavefunction and the capability of improving the nodes during the optimization is crucial to obtain almost exact DMC energy values. To that purpose, it is very important to have a variational functional form appropriate to reproduce the correct nodes. We show that the $A G P+J$ wavefunction satisfies well this requirement, yielding in all the atoms studied here very good DMC results. The VMC and DMC energies are listed in Table \ in Figures 1 and 2 we plot the percentage of the correlation energy recovered respectively by VMC and DMC calculations for different atoms and wavefunctions.

The VMC calculations with the minimal geminal expansion and the two body Jastrow factor yield from $60 \%$ to $68 \%$ of the total correlation energy, with the exception of the $L i$ atom, where $91.4 \%$ of the correlation energy is obtained. Therefore, there is a sizable loss of accuracy in going from $L i$ to $B e$, the worst case being the Boron atom. The corresponding DMC simulations get a large amount of the energy missing in the VMC calculations, recovering from $87.7 \%$ to $99.9 \%$ of the total correlation energy, but the dependence on the atomic number shows the same behavior found in VMC: the worst results are obtained for $B e, B$ and $C$ atoms, due to the strong multiconfigurational nature of their ground states. 
As well known, one can improve substantially the variational state of those atoms including not only the $1 s^{2} 2 s^{2}$ configuration but also the $2 s^{2} 2 p^{2}$, because of the near degeneracy of $2 s$ and $2 p$ orbitals. In this case the $A G P+J$ ansatz is particularly efficient: by adding just one term in the geminal expansion, we are able to remove this loss of accuracy in the correlation energy both in the VMC and the DMC calculations.

In Table III we summarize some results obtained for $B e$ in previous works and compare them with ours. AGP calculations of atoms have been performed only few times so far, the best one for $B e$ is reported in the last row of the Table [1] Kurtz et al. 27] were able to recover $84 \%$ of correlation energy using a geminal expansion with a very large basis; our variational $A G P+J$ wavefunction reaches $94 \%$ with a much smaller basis $(1 s, 2 s$ and $2 p$ orbitals). By including a three-body Jastrow factor, $97.5 \%$ of the correlation energy is finally obtained, which is comparable with the best multiconfigurational wavefunctions previously studied [24].

This outcome highlights the importance of the Jastrow in reducing the geminal expansion and yielding a better energy. The nodal surface can be substantially improved with the present approach, because the pairing expansion contains implicitly not only the four determinants $1 s^{2} 2 s^{2}$ and $1 s^{2} 2 p^{2}$, but also the other three $2 s^{2} 2 p^{2}$ and six $2 p^{2} 2 p^{2}$, which can improve further the wavefunction. Indeed, the geminal expansion reduces exactly to four determinants in the limit $\lambda_{2 s} \rightarrow 0$ and $\lambda_{2 p} \rightarrow 0$ with constant ratio $\frac{\lambda_{2 s}}{\lambda_{2 p}}$. The fact that the minimum energy is obtained for $\lambda_{2 s} \neq 0$ and $\lambda_{2 p} \neq 0$ (see Tables IV and $\nabla$ ) clearly shows that the energy can be lowered by considering the remaining configurations described above. Indeed our DMC energies are slightly lower than the ones by Huang et al. [24], to our knowledge the best available ones obtained with the four determinants: one $1 s^{2} 2 s^{2}$ and three $1 s^{2} 2 p^{2}$. In order to determine accurate nodes for the corresponding DMC calculation they used the two-body Jastrow factor similar to the one (18) we used or an highly involved three-body term much more complete than ours (for this reason our corresponding VMC energy is slightly worse in this case). We also verified, therefore, that a more accurate description of the Jastrow factor (which do not affect directly the nodes) is crucial to obtain better nodes, provided the variational parameters, belonging to both the Jastrow and the determinantal part, are optimized altogether. For instance, in Ref. 22 the authors optimized only the coefficients in front of the four determinants $1 s 2 s-1 s 2 p$ and not the orbitals, obtaining for $B e$ energy not comparable with the best possible ones. The $A G P+J$ is simple enough to allow a feasible parametrization of the variational state, by capturing the most important correlation.

We found that also $M g, A l$ and $S i$, the second row atoms corresponding to $B e, B$ and $C$ in the first row, have a quite strong multiconfigurational character, involving here $3 s$ and $3 p$ orbitals. Analogously to the Beryllium case, for the $M g$ we have optimized both the two-body and the three-body Jastrow factor, together with the $A G P$ wavefunction containing $3 s-3 p$ resonance. In this case, although at the variational level the threebody wavefunction is much better than the two-body one (see Fig. 1), that difference disappears almost completely in the DMC results. This shows that the correction of the nodal surface allowed by the more accurate threebody Jastrow does not seem to be crucial as in the $B e$ atom. On the other hand, the effect of the $A G P$ expansion is significant in improving further the DMC calculation, which already yields good FN energies even with a simple $H F+J$ trial wavefunction for atoms heavier than $C$ (percentage of correlation energy always greater than $92 \%)$. By adding the $3 p$ contribution to the geminal we were able to recover $96.8 \%$ of the correlation energy of $M g$ (see Fig. 2). Also for $A l$ the presence of the $3 p$ orbital is significant in reducing the DMC energy, and for $S i$ it seems important only in the VMC value.

Finally, by using the $A G P+J$ wavefunction, we optimized some atoms (from $N$ to $N a$ ) not affected by nondynamic correlation; here, in order to obtain an improvement in the VMC and in the DMC energies, we needed a bigger basis $(3 s 2 p 1 d)$ to be used in the geminal expansion.

\section{CONCLUSIONS AND PERSPECTIVES}

In this work we have introduced a variational wavefunction which contains the main ingredients of electron correlations: the Jastrow factor, that allows to satisfy the electron-electron cusp condition, and the geminal expansion, that allows to consider a correlated multiconfiguration state, with a numerically feasible scheme, namely by evaluating only a single but appropriately defined determinant.

The application to atoms is particularly successful for low atomic number, where Hartree-Fock is particularly poor, due to the almost degenerate $2 s-2 p$ shells. The case of Beryllium is an interesting benchmark. Indeed, by considering the change of the geminal part altogether with the Jastrow term, we obtained an excellent representation of this correlated atom. Our results, presented in Table III are not only comparable but appear even better than the best multideterminantal schemes (using e.g. four Slater determinants), showing that it is possible to represent non trivial correlated states by properly taking into account the interplay of the Jastrow term and the determinantal part of the wavefunction. Our variational energies for the other atoms (see Table I) can be substantially lowered because we have considered, in this first application, a wavefunction with the two-body Jastrow factor.

As well known the variational energy of the HartreeFock wavefunction cannot be improved by extending the variational calculation to a larger basis including all particle-hole excitations applied to the Hartree-Fock state. Analogously, the geminal wavefunction is not only 
stable with respect to these particle-hole configurations , but also to all possible states obtained by destroying a singlet pair on some orbital and creating another one on another orbital. Though this wavefunction can take into account a big number of configurations which may allow an energy improvement, obviously it cannot include everything within a single geminal, otherwise the wavefunction would be exact. Indeed there exist multiconfigurational states that are known to be important for atoms like $C$ or those with larger $Z$ [29], and that involve complicated multi-particle excitations to the Hartree-Fock state. These ones cannot be reduced to creation/destruction of singlet pairs and therefore cannot be handled with a single geminal function. However in our study we have found that the single geminal function with the proper Jastrow factor already provides satisfactory results for all atoms, yielding more than $93 \%$ of the correlation energy in all cases studied by carrying out DMC simulations.

The extension of this approach to molecules or more complex electronic systems is straightforward, and is indeed particularly promising. As pointed out in Ref. 28, the geminal wavefunction for a diatomic system can correctly describe the interatomic Born-Oppenheimer potential from small to large distances, where, in this limit, the wavefunction of isolated atoms can be smoothly recovered. This important property cannot be satisfied within the Hartree-Fock theory, even for the simplest $\mathrm{H}_{2}$ molecule (without contaminating the singlet ground state wavefunction).

For an electronic system with many atoms, the geminal expansion together with the Jastrow term is very similar to the so called Resonating Valence Bond (RVB) expansion, introduced by Pauling (see e.g. 30]) long time ago, and revived recently by P.W. Anderson to consider the properties of strongly correlated electronic systems. The geminal part, when expanded in terms of Slater determinants, yields a very large and non trivial number of configurations, which increases exponentially with the number of atoms considered. The Jastrow factor in this case suppresses the weight of those configurations with two electrons close to the same atomic orbital, correctly describing the effect of the strong Coulomb repulsion. We see therefore the remarkable advantage of this approach. Just for complex systems with many atoms an RVB wavefunction corresponding to an exponentially large number of configurations can be efficiently used for a more accurate description of electron correlation. Contrary to the conventional RVB expansion, it is appealing, not only from the computational point of view, that these properties can be obtained by sampling a single determinant wavefunction within the Quantum Monte Carlo techniques.

\section{Acknowledgments}

We gratefully acknowledge Saverio Moroni, for having provided us unpublished work on the Neon atom, and Claudia Filippi for useful comments on the preliminary version of the manuscript. We also thank G. Bachelet, F. Becca and C. Attaccalite for many useful discussions. This work was partially supported by MIUR-COFIN 2001.

\section{APPENDIX: SPIN POLARIZED GEMINAL WAVEFUNCTION}

In this appendix, we consider the most general geminal wavefunction with definite spin $S=\frac{N_{\uparrow}-N_{\downarrow}}{2}$, where $N_{\uparrow}\left(N_{\downarrow}\right)$ is the number of spin-up (spin-down) electrons and $N_{\uparrow}>N_{\downarrow}$ is assumed. To this purpose we introduce second quantized fermionic fields (see e.g. Fetter and Walecka 31] $\psi^{\dagger}(\mathbf{r}, \sigma)$ and $\psi(\mathbf{r}, \sigma)$, where $\mathbf{r}$ is the electron position and $\sigma= \pm 1 / 2$ is its spin projection along the $z$-axis. These fields satisfy the canonical anticommutation rules:

$$
\left\{\psi(\mathbf{r}, \sigma), \psi^{\dagger}\left(\mathbf{r}^{\prime}, \sigma^{\prime}\right)\right\}=\delta_{\sigma \sigma^{\prime}} \delta\left(\mathbf{r}-\mathbf{r}^{\prime}\right) .
$$

In these notations, the most general wavefunction with definite spin can be formally written in the following way:

$$
|\Psi\rangle=P_{N} \prod_{i=N_{\downarrow}+1}^{N_{\uparrow}} \psi_{i, \uparrow}^{\dagger} \exp \left(\Phi^{\dagger}\right)|0\rangle,
$$

where $P_{N}$ is the projection on the given number of particles $N=N_{\uparrow}+N_{\downarrow},|0\rangle$ denotes the vacuum of electrons and $\psi_{i, \uparrow}^{\dagger}$ is the most generic (Bogoliubov) orbital with $\operatorname{spin} S=1 / 2$ :

$$
\psi_{i, \uparrow}^{\dagger}=\int d \mathbf{r}\left(\phi_{i}^{<}(\mathbf{r}) \psi(\mathbf{r}, \downarrow)+\phi_{i}^{>}(\mathbf{r}) \psi^{\dagger}(\mathbf{r}, \uparrow)\right),
$$

which is defined by the orbital functions $\phi_{i}^{>}$for the creation of a particle with spin up and $\phi_{i}^{<}$for the annihilation of a particle with spin down. For instance, a conventional Slater determinant of spin-up particles can be written as $\prod_{i} \psi_{i, \uparrow}^{\dagger}|0\rangle$, where $\phi_{i}^{<}=0$. It is clear therefore that this representation is more general and may provide a wavefunction $\Psi$ much more reach than the conventional Slater determinants.

Finally, the pairing creation operator $\Phi^{\dagger}$ is a singlet, namely $\exp \left(\Phi^{\dagger}\right)|0\rangle$ has spin zero, and is defined by a generic symmetric function $\Phi\left(\mathbf{r}, \mathbf{r}^{\prime}\right)=\Phi\left(\mathbf{r}^{\prime}, \mathbf{r}\right)$ :

$$
\Phi^{\dagger}=\int d \mathbf{r} \int d \mathbf{r}^{\prime} \Phi\left(\mathbf{r}^{\prime}, \mathbf{r}\right) \psi^{\dagger}(\mathbf{r}, \downarrow) \psi^{\dagger}\left(\mathbf{r}^{\prime}, \uparrow\right) .
$$

Our purpose is to show here that the value of the wavefunction $\Psi$ can be simply computed, similarly to 
a conventional Slater determinant, on each configuration $x=\left\{\mathbf{r}_{1, \uparrow}, \ldots, \mathbf{r}_{N_{\downarrow}, \downarrow}\right\}$, where $\mathbf{r}_{i, \uparrow}$ are the positions of spinup particles and $\mathbf{r}_{i, \downarrow}$ are the spin-down ones. These configurations can be generally written as:

$$
\langle x|=\langle 0| \prod_{i=1}^{N_{\uparrow}} \psi\left(\mathbf{r}_{i}, \uparrow\right) \prod_{j=1}^{N_{\downarrow}} \psi\left(\mathbf{r}_{j}, \downarrow\right) .
$$

Indeed the value $F$ of the wavefunction on $\langle x|$ is:

$$
F=\langle x \mid \Psi\rangle=\left\langle 0\left|\prod_{i} \psi\left(\mathbf{r}_{i}, \uparrow\right) \prod_{j} \psi\left(\mathbf{r}_{j}, \downarrow\right) \prod_{k=N_{\downarrow}+1}^{N_{\uparrow}} \psi_{k, \uparrow}^{\dagger} \exp \left(\Phi^{\dagger}\right)\right| 0\right\rangle .
$$

Now we insert the identity $\exp \left(-\Phi^{\dagger}\right) \exp \left(\Phi^{\dagger}\right)$ between each fermionic field in the above equation (A.6):

$$
\begin{aligned}
F=\langle & \langle 0| \exp \left(\Phi^{\dagger}\right) \exp \left(-\Phi^{\dagger}\right) \psi\left(\mathbf{r}_{1}, \uparrow\right) \exp \left(\Phi^{\dagger}\right) \cdots \\
& \cdots \exp \left(-\Phi^{\dagger}\right) \psi\left(\mathbf{r}_{N_{\downarrow}}, \downarrow\right) \exp \left(\Phi^{\dagger}\right) \cdots \\
& \cdots \exp \left(-\Phi^{\dagger}\right) \psi_{N_{\uparrow}, \uparrow}^{\dagger} \exp \left(\Phi^{\dagger}\right)|0\rangle
\end{aligned}
$$

Exploiting the relation valid for generic operators $A, B$ and $C$ :

$$
\exp (-A) B \exp (A)=B-[A, B]+\frac{1}{2}[A,[A, B]]+\ldots
$$

one is able to evaluate the following terms:

$$
\begin{array}{r}
\exp \left(-\Phi^{\dagger}\right) \psi\left(\mathbf{r}_{i}, \uparrow\right) \exp \left(\Phi^{\dagger}\right)= \\
\psi\left(\mathbf{r}_{i}, \uparrow\right)-\int d \mathbf{r} \Phi\left(\mathbf{r}_{i, \uparrow}, \mathbf{r}\right) \psi^{\dagger}(\mathbf{r}, \downarrow) \\
\exp \left(-\Phi^{\dagger}\right) \psi\left(\mathbf{r}_{i}, \downarrow\right) \exp \left(\Phi^{\dagger}\right)= \\
\psi\left(\mathbf{r}_{i}, \downarrow\right)+\int d \mathbf{r} \Phi\left(\mathbf{r}, \mathbf{r}_{i, \downarrow}\right) \psi^{\dagger}(\mathbf{r}, \uparrow) \\
\exp \left(-\Phi^{\dagger}\right) \psi_{i, \uparrow}^{\dagger} \exp \left(\Phi^{\dagger}\right)= \\
\psi_{i, \uparrow}^{\dagger}+\int d \mathbf{r} \int d \mathbf{r}^{\prime} \Phi\left(\mathbf{r}, \mathbf{r}^{\prime}\right) \phi_{i}^{<}\left(\mathbf{r}^{\prime}\right) \psi^{\dagger}(\mathbf{r}, \uparrow)
\end{array}
$$

In order to derive the above relations, notice that all the terms in the RHS of Eq. A.8 are always zero beyond the first two. After substituting the expressions in Eq. A.7 and by using $\langle 0| \exp \left(\Phi^{\dagger}\right)=\langle 0|, \psi(\mathbf{r}, \sigma)| 0\rangle=0$ and $\langle 0| \psi^{\dagger}(\mathbf{r}, \sigma)=0$, one can iteratively apply the canonical commutation rules A.1 and a simplified result for $F$ is obtained:

$$
F=\left\langle 0\left|\prod_{i=1}^{N_{\uparrow}} \psi\left(\mathbf{r}_{i}, \uparrow\right) \prod_{i=1}^{N_{\uparrow}} \tilde{\psi}_{i, \uparrow}^{\dagger}\right| 0\right\rangle
$$

where $\tilde{\psi}_{i, \uparrow}^{\dagger}$ is the creator of an orbital function of the type A.3), with transformed orbitals:

$$
\begin{aligned}
\phi_{i}(\mathbf{r})= & \Phi\left(\mathbf{r}, \mathbf{r}_{i, \downarrow}\right) \\
& \text { for } i=1, \cdots, N_{\downarrow} \\
\bar{\phi}_{i}(\mathbf{r})= & \phi_{i}^{>}(\mathbf{r})+\int d \mathbf{r}^{\prime} \Phi\left(\mathbf{r}, \mathbf{r}^{\prime}\right) \phi_{i}^{<}\left(\mathbf{r}^{\prime}\right) \\
& \text { for } i=N_{\downarrow}+1, \cdots, N_{\uparrow}
\end{aligned}
$$

Then the final value of $F$ can be simply computed by a single determinant, as it represents just the value of a $N_{\uparrow} \times N_{\uparrow}$ Slater determinant with orbitals given in A.11a and A.11b on the spin-up configurations, yielding the final expression (66) reported in the text.
[1] A. C. Hurley, J. E. Lennard-Jones, and J. A. Pople, Proc. R. Soc. London, Ser. A 220, 446 (1953)

[2] J. R. Schrieffer in The Theory of Superconductivity, 5th printing (Addison-Wesley, 1994)

[3] S. Bratoz and P. Durand, J. Chem. Phys. 43, 2670 (1965)

[4] B. Weiner and O. Goscinski, Phys. Rev. A 22, 2374 (1980)

[5] G. Bessis, C. Murez and S. Bratoz, Int. J. Quantum Chem.1, 327 (1967)

[6] H. A. Kurtz and N. Elander, Int. J. Quantum Chem. Quantum Chem. Symp. 16, 605 (1982)
[7] C. J. Umrigar in Quantum Monte Carlo Methods in Physics and Chemistry, Proceedings of the NATO Advanced Study Institute, edited by M. P. Nightingale and C. J. Umrigar (Kluwer, Dordrecht, 1998), page 129

[8] P. W. Anderson, Mat. Res. Bull. 8, 153 (1973)

[9] P. W. Anderson, Science 235, 1196 (1987)

[10] J. P. Bouchaud and C. Lhuillier, Z. Phys. B 75, 283 (1989)

[11] S. Sorella, Phys. Rev. B 64, 024512 (2001)

[12] A. Harju, B. Barbiellini, S. Siljamaki, R.M. Nieminen and G. Ortiz, Phys. Rev. Lett. 79, 1173 (1997) 
[13] S. J. Chakravorty, S. R. Gwaltney, E. R. Davidson, F. A. Parpia and C. F. Fischer, Phys. Rev. A 47, 3649 (1993)

[14] J. P. Bouchaud, AS. Georges, C. Lhuillier, J. Phys. (Paris) 49, 553 (1988)

[15] A. J. Coleman, J. Math. Phys. 13, 214 (1972)

[16] P. O. Löwdin and H. Shull, Phys. Rev. 101, 1730 (1956)

[17] E. Clementi and C. Roetti, Atomic Data and Nuclear Data Tables (Academic Press, 1974)

[18] T. Kato, Commun. Pure Appl. Math. 10, 151 (1957)

[19] R. T. Pack and W. B. Brown, J. Chem. Phys. 45, 556 (1966)

[20] C. Filippi and C. J. Umrigar, J. Chem. Phys. 105, 213 (1996)

[21] C. J. Umrigar, K. G. Wilson and J. W. Wilkins, Phys. Rev. Lett. 60, 1719 (1988)

[22] A. Sarsa, F. J. Gálvez and E. Buendía, J. Chem. Phys. 109, 7075 (1998)

[23] Heinz-Jürgen Flad and A. Savin, J. Chem. Phys. 103, 691 (1995)

[24] Chien-Jung Huang, C. J. Umrigar and M. P. Nightingale,
J. Chem Phys. 107, 3007 (1997)

[25] K. E. Schmidt and J. W. Moskowitz, J. Chem. Phys. 93, 4172 (1990)

[26] C. Filippi and S. Fahy, J. Chem. Phys. 112, 3523 (2000)

[27] H. A. Kurtz, N. Elander, O. Goscinski and E. Sangfelt, Int. J. Quantum Chem. Quantum Chem. Symp. 15, 143 (1981)

[28] V. A. Rassolov, J. Chem. Phys. 117, 5978 (2002)

[29] Heinz-Jürgen Flad, M. Caffarel and A. Savin in Recent Advances in Quantum Monte Carlo Methods, edited by W. A. Lester (World Scientific Publishing, Singapore 1997), page 73

[30] L. Pauling in The nature of the chemical bond and structure of molecules and crystals: an introduction to modern structural chemistry (Cornell University Press, Ithaca, New York, 1960)

[31] A. L. Fetter and J. D. Walecka in Quantum theory of many-particle systems (McGraw-Hill, New York, 1971) 
TABLE I: Total energies in variational $\left(E_{V M C}\right)$ and diffusion $\left(E_{D M C}\right)$ Monte Carlo calculations; the percentages of correlation energy recovered in $\mathrm{VMC}\left(E_{c}^{V M C}(\%)\right)$ and DMC $\left(E_{c}^{D M C}(\%)\right)$ have been evaluated using the exact $\left(E_{0}\right)$ and Hartree-Fock $\left(E_{H F}\right)$ energies from Ref 13 . $M$ is the number of terms in the pairing expansion. The energies are in Hartree.

\begin{tabular}{|c|c|c|c|c|c|c|c|}
\hline & $M$ & $\overline{E_{0}}$ & $E_{H F}$ & $E_{V M C}$ & $E_{c}^{V M C}(\%)$ & $E_{D M C}$ & $E_{c}^{D M C}(\%)$ \\
\hline $\mathrm{Li}$ & 1 & -7.47806 & -7.432727 & $-7.47415(10)$ & $91.4(2)$ & $-7.4780(2)$ & $99.9(4)$ \\
\hline \multirow[t]{3}{*}{$\mathrm{Be}$} & 2 & -14.66736 & -14.573023 & $-14.63145(5)$ & $61.9(5)$ & $-14.6565(4)$ & $88.5(4)$ \\
\hline & 5 & & & $-14.661695(10)$ & $93.995(11)$ & $-14.66711(3)$ & $99.73(3)$ \\
\hline & $5^{a}$ & & & $-14.66504(4)$ & $97.54(5)$ & $-14.66728(2)$ & $99.92(2)$ \\
\hline \multirow[t]{2}{*}{ B } & 2 & -24.65391 & -24.529061 & $-24.6042(3)$ & $60.3(2)$ & $-24.63855(5)$ & $87.7(4)$ \\
\hline & 5 & & & $-24.62801(4)$ & $79.25(4)$ & $-24.6493(3)$ & $96.3(3)$ \\
\hline \multirow[t]{2}{*}{$\mathrm{C}$} & 2 & -37.8450 & -37.688619 & $-37.7848(6)$ & $61.5(4)$ & $-37.8296(8)$ & $90.2(5)$ \\
\hline & 5 & & & $-37.7985(7)$ & $70.3(4)$ & $-37.8359(8)$ & $94.2(5)$ \\
\hline \multirow[t]{3}{*}{$\mathrm{N}$} & 2 & -54.5892 & -54.400934 & $-54.52180(15)$ & $64.20(8)$ & $-54.57555(5)$ & $92.7(3)$ \\
\hline & $2^{b}$ & & & $-54.52565(15)$ & $66.20(8)$ & $-54.5753(4)$ & $92.6(2)$ \\
\hline & 14 & & & $-54.5263(2)$ & $66.62(11)$ & $-54.5769(2)$ & $93.47(10)$ \\
\hline \multirow[t]{2}{*}{$\mathrm{O}$} & 3 & -75.0673 & -74.809398 & $-74.9750(7)$ & $64.2(3)$ & $-75.0477(8)$ & $92.4(3)$ \\
\hline & 14 & & & $-74.9838(6)$ & $67.6(2)$ & $-75.0516(9)$ & $93.9(3)$ \\
\hline \multirow[t]{2}{*}{$\mathrm{F}$} & 4 & -99.7339 & -99.409349 & $-99.6190(8)$ & $64.6(3)$ & $-99.7145(15)$ & $94.0(5)$ \\
\hline & 14 & & & $-99.6315(7)$ & $68.4(2)$ & $-99.7141(6)$ & $93.91(18)$ \\
\hline \multirow[t]{2}{*}{$\mathrm{Ne}$} & 5 & -128.9376 & -128.547098 & $-128.8070(10)$ & $66.6(3)$ & $-128.9204(8)$ & $95.6(2)$ \\
\hline & 14 & & & $-128.8159(6)$ & $68.83(17)$ & $-128.9199(7)$ & $95.47(18)$ \\
\hline \multirow[t]{2}{*}{$\mathrm{Na}$} & 5 & -162.2546 & -161.858912 & $-162.1334(7)$ & $69.37(19)$ & $-162.2325(10)$ & $94.4(2)$ \\
\hline & 9 & & & $-162.1434(7)$ & $71.91(16)$ & $-162.2370(10)$ & $95.5(2)$ \\
\hline \multirow[t]{3}{*}{$\mathrm{Mg}$} & 6 & -200.053 & -199.614636 & $-199.9113(8)$ & $67.67(19)$ & $-200.0327(9)$ & $95.4(2)$ \\
\hline & 9 & & & $-199.9363(8)$ & $73.38(19)$ & $-200.0375(10)$ & $96.5(2)$ \\
\hline & $9^{a}$ & & & $-200.0002(5)$ & $87.95(12)$ & $-200.0389(5)$ & $96.79(11)$ \\
\hline \multirow[t]{2}{*}{$\mathrm{Al}$} & 6 & -242.346 & -241.876707 & $-242.1900(9)$ & $66.77(19)$ & $-242.3215(10)$ & $94.8(2)$ \\
\hline & 9 & & & $-242.2124(9)$ & $71.53(19)$ & $-242.3265(10)$ & $95.8(2)$ \\
\hline \multirow[t]{2}{*}{$\mathrm{Si}$} & 6 & -289.359 & -288.854363 & $-283.1875(10)$ & $66.0(2)$ & $-289.3275(10)$ & $93.8(2)$ \\
\hline & 9 & & & $-289.1970(10)$ & $67.9(2)$ & $-289.3285(10)$ & $94.0(2)$ \\
\hline $\mathrm{P}$ & 6 & -341.259 & -340.718781 & $-341.0700(10)$ & $65.0(2)$ & $-341.2220(15)$ & $93.2(3)$ \\
\hline
\end{tabular}

${ }^{a}$ Wavefunction with a three body Jastrow factor.

${ }^{b}$ Wavefunction with a two body Jastrow factor spin contaminated.

TABLE II: Comparison of the energies obtained by various authors for $B e$.

\begin{tabular}{ccccc}
\hline \hline & basis & Jastrow & VMC & DMC \\
\hline present work & $2 \mathrm{~s} 1 \mathrm{p}$ & two body & $-14.661695(10)$ & $-14.66711(3)$ \\
Huang et al. $[24]$ & $2 \mathrm{~s} 1 \mathrm{p}$ & two body & $-14.66088(5)$ & $-14.66689(4)$ \\
present work & $2 \mathrm{~s} 1 \mathrm{p}$ & three body & $-14.66504(4)$ & $-14.66728(2)$ \\
Huang ETA. $[24]$ & $2 \mathrm{~s} 1 \mathrm{p}$ & three body & $-14.66662(1)$ & $-14.66723(1)$ \\
Sarsa et al. $[22]$ & $2 \mathrm{~s} 1 \mathrm{p}$ & three body & $-14.6523(1)$ & \\
Kurtz et al. $[27]$ & $6 \mathrm{~s} 3 \mathrm{p} 2 \mathrm{~d}$ & none & -14.6171 & \\
\hline \hline
\end{tabular}


TABLE III: $\mathbf{H F}+\mathbf{J}$ (two body) wavefunctions

Parameters of the Jastrow and the pairing function with the notation described in the text. "\#" means that the corresponding parameter has to be evaluated from the cusp condition in Eq. 15] The line over the orbitals label refers to the unpaired ones. The values are given with the statistic error due to the stochastic approach in the minimization.

\begin{tabular}{|c|c|c|c|c|c|c|}
\hline & $b$ & orbital & $Z_{1}$ & $Z_{2}$ & $p$ & $\lambda$ \\
\hline \multirow[t]{2}{*}{$\overline{\mathrm{Li}}$} & \multirow{2}{*}{$0.731(3)$} & $1 s$ & $3.556(2)$ & $2.3741(15)$ & $\#$ & 1.0 \\
\hline & & $\overline{2 s}$ & $1.4289(12)$ & $0.5380(2)$ & $\#$ & \\
\hline \multirow[t]{2}{*}{$\overline{\mathrm{Be}}$} & \multirow[t]{2}{*}{$0.773(2)$} & $1 s$ & $4.569(6)$ & $3.289(5)$ & $\#$ & 1.0 \\
\hline & & $2 s$ & $2.602(3)$ & $0.7850(8)$ & \# & 1.0 \\
\hline \multirow[t]{3}{*}{$\overline{\mathrm{B}}$} & \multirow[t]{3}{*}{$0.877(2)$} & $1 s$ & $5.569(5)$ & $4.195(4)$ & $\#$ & 1.0 \\
\hline & & $2 s$ & $3.527(2)$ & $1.0788(2)$ & \# & 1.0 \\
\hline & & $\overline{2 p}$ & $2.437(4)$ & $1.1001(5)$ & $0.2664(3)$ & \\
\hline \multirow[t]{3}{*}{$\overline{\mathrm{C}}$} & \multirow[t]{3}{*}{$0.990(2)$} & $1 s$ & $6.533(6)$ & $5.075(8)$ & $\#$ & 1.0 \\
\hline & & $2 s$ & $4.475(3)$ & $1.3552(3)$ & $\#$ & 1.0 \\
\hline & & $\overline{2 p}$ & $2.9835(12)$ & $1.3886(5)$ & $0.2374(4)$ & \\
\hline \multirow[t]{3}{*}{$\mathrm{N}$} & \multirow[t]{3}{*}{$1.110(3)$} & $1 s$ & $7.461(8)$ & $5.901(15)$ & $\#$ & 1.0 \\
\hline & & $2 s$ & $5.387(3)$ & $1.6323(1)$ & $\#$ & 1.0 \\
\hline & & $\overline{2 p}$ & $3.4908(9)$ & $1.6373(4)$ & $0.2036(3)$ & \\
\hline \multirow[t]{3}{*}{$\overline{\mathrm{N}}$} & \multirow{3}{*}{$\begin{array}{c}\text { contaminated } \\
b_{\uparrow \downarrow}=0.940(3) \\
b_{\uparrow \uparrow}=0.624(4)\end{array}$} & $1 s$ & $7.2738(17)$ & $5.522(7)$ & $\#$ & 1.0 \\
\hline & & $2 s$ & $5.254(2)$ & $1.6692(15)$ & $\#$ & 1.0 \\
\hline & & $\overline{2 p}$ & $3.4544(4)$ & $1.66559(13)$ & $0.21593(9)$ & \\
\hline \multirow[t]{4}{*}{$\overline{\mathrm{O}}$} & \multirow[t]{4}{*}{$1.152(2)$} & $1 s$ & $8.303(5)$ & $6.53(3)$ & $\#$ & 1.0 \\
\hline & & $2 s$ & $6.428(2)$ & $1.9362(5)$ & $\#$ & 1.0 \\
\hline & & $2 p$ & $3.9921(7)$ & $1.84932(15)$ & $0.1648(2)$ & 1.0 \\
\hline & & $\frac{-T}{2 p}$ & $3.9921(7)$ & $1.84932(15)$ & $0.1648(2)$ & \\
\hline \multirow[t]{4}{*}{$\mathrm{F}$} & \multirow[t]{4}{*}{$1.226(9)$} & $1 s$ & $9.171(3)$ & $6.82(2)$ & $\#$ & 1.0 \\
\hline & & $2 s$ & $7.380(2)$ & $2.2402(4)$ & \# & 1.0 \\
\hline & & $2 p$ & $4.5025(2)$ & $2.0758(2)$ & $0.1451(10)$ & 1.0 \\
\hline & & $\frac{1}{2 p}$ & $4.5025(2)$ & $2.0758(2)$ & $0.1451(10)$ & \\
\hline \multirow[t]{3}{*}{$\mathrm{Ne}$} & \multirow[t]{3}{*}{$1.321(3)$} & $1 s$ & $10.103(4)$ & $7.01(4)$ & $\#$ & 1.0 \\
\hline & & $2 s$ & $8.341(10)$ & $2.5319(5)$ & $\#$ & 1.0 \\
\hline & & $2 p$ & $5.0273(10)$ & $2.3155(4)$ & $0.1358(2)$ & 1.0 \\
\hline \multirow[t]{4}{*}{$\mathrm{Na}$} & \multirow[t]{4}{*}{$1.514(7)$} & $1 s$ & $11.102(2)$ & $7.58(5)$ & $\#$ & 1.0 \\
\hline & & $2 s$ & $8.823(2)$ & $2.8795(4)$ & $\#$ & 1.0 \\
\hline & & $2 p$ & $5.7178(9)$ & $2.8092(8)$ & $0.18027(18)$ & 1.0 \\
\hline & & $\frac{1}{3 s}$ & $1.540(3)$ & $0.734(2)$ & $0.1265(2)$ & \\
\hline $\mathrm{Mg}$ & $1.654(6)$ & $1 s$ & $12.0855(15)$ & $7.96(5)$ & \# & 1.0 \\
\hline & & $2 s$ & $9.349(4)$ & $3.2611(16)$ & \# & 1.0 \\
\hline & & $2 p$ & $6.3795(10)$ & $3.2905(5)$ & $0.21569(17)$ & 1.0 \\
\hline & & $3 s$ & $1.9288(10)$ & $1.02214(13)$ & $0.1449(2)$ & 1.0 \\
\hline$\overline{\mathrm{Al}}$ & $1.812(8)$ & $1 s$ & $13.0791(17)$ & $8.43(7)$ & $\#$ & 1.0 \\
\hline & & $2 s$ & $9.846(8)$ & $3.646(2)$ & \# & 1.0 \\
\hline & & $2 p$ & $7.0560(15)$ & $3.7873(7)$ & $0.2597(3)$ & 1.0 \\
\hline & & $3 s$ & $2.24(8)$ & $1.2627(2)$ & $0.1445(2)$ & 1.0 \\
\hline & & $\overline{3 p}$ & $1.83(2)$ & $0.8866(2)$ & $0.1012(7)$ & \\
\hline $\mathrm{Si}$ & $1.961(7)$ & $1 s$ & $14.072(2)$ & $8.77(8)$ & $\#$ & 1.0 \\
\hline & & $2 s$ & $10.40(5)$ & $4.0275(8)$ & \# & 1.0 \\
\hline & & $2 p$ & $7.703(10)$ & $4.261(5)$ & $0.2944(12)$ & 1.0 \\
\hline & & $3 s$ & $2.468(5)$ & $1.46(2)$ & $0.1241(2)$ & 1.0 \\
\hline & & $\overline{3 p}$ & $2.274(2)$ & $1.16(2)$ & $0.1227(3)$ & \\
\hline$\overline{\mathrm{P}}$ & $2.074(9)$ & $1 s$ & $15.053(4)$ & $9.10(15)$ & $\#$ & 1.0 \\
\hline & & $2 s$ & $10.997(5)$ & $4.4269(5)$ & \# & 1.0 \\
\hline & & $2 p$ & $8.346(2)$ & $4.7519(12)$ & $0.3273(17)$ & 1.0 \\
\hline & & $3 s$ & $2.7386(5)$ & $1.62(2)$ & $0.1129(3)$ & 1.0 \\
\hline & & $\overline{3 p}$ & $2.5918(18)$ & $1.3479(7)$ & $0.1125(2)$ & \\
\hline
\end{tabular}


TABLE IV: AGP+J (two body) wavefunctions The notations are the same as in Table III

\begin{tabular}{|c|c|c|c|c|c|c|}
\hline & $b$ & orbital & $Z_{1}$ & $Z_{2}$ & $p$ & $\lambda$ \\
\hline \multirow[t]{3}{*}{$\overline{\mathrm{Be}}$} & $1.009(5)$ & $1 s$ & $4.7139(6)$ & $3.3118(3)$ & $\#$ & 1.0 \\
\hline & & $2 s$ & $2.27561(16)$ & $0.78955(2)$ & $\#$ & $-1.95(8) 10^{-3}$ \\
\hline & & $2 p$ & $3.389(2)$ & $1.06495(4)$ & $0.8878(18)$ & $3.49(15) 10^{-4}$ \\
\hline \multirow{3}{*}{$\overline{\mathrm{B}}$} & & $2 s$ & $3.35545(19)$ & $1.07294(2)$ & $\#$ & $-4.41(7) 10^{-3}$ \\
\hline & & $2 p$ & $3.701(7)$ & $1.46298(13)$ & $0.827(2)$ & $6.92(10) 10^{-4}$ \\
\hline & & $\frac{i}{2 p}$ & $2.4093(3)$ & $1.07197(4)$ & $0.28102(8)$ & \\
\hline \multirow{3}{*}{$\mathrm{C}$} & & $2 s$ & $4.4094(3)$ & $1.353943(18)$ & $\#$ & $-5.40(10) 10^{-3}$ \\
\hline & & $2 p$ & $4.289(3)$ & $1.8817(2)$ & $0.7769(8)$ & $7.53(14) 10^{-4}$ \\
\hline & & $\frac{i}{2 p}$ & $2.95758(8)$ & $1.36288(2)$ & $0.23276(2)$ & \\
\hline \multirow[t]{3}{*}{$\mathrm{N}$} & $1.124(5)$ & $1 s$ & $7.4553(11)$ & $5.866(2)$ & $\#$ & 1.0 \\
\hline & & $2 s$ & $5.5031(12)$ & $1.61305(8)$ & $\#$ & $-2.345(9) 10^{-2}$ \\
\hline & & $2 p$ & $5.708(9)$ & $2.5488(8)$ & $0.9834(14)$ & $2.538(9) 10^{-3}$ \\
\hline \multirow[t]{7}{*}{$\mathrm{O}$} & $1.2073(10)$ & $1 s$ & $8.3359(4)$ & $6.5514(15)$ & $\#$ & 1.0 \\
\hline & & $2 s$ & $6.4248(3)$ & $1.94127(5)$ & \# & $-9.54(6) 10^{-3}$ \\
\hline & & $2 p$ & $3.97418(5)$ & $1.77990(4)$ & $0.168724(8)$ & $-5.73(4) 10^{-3}$ \\
\hline & & $3 s$ & $5.3337(16)$ & & $0.0^{a}$ & $3.09(2) 10^{-4}$ \\
\hline & & $3 p$ & $2.00849(8)$ & & $0.0^{a}$ & $2.156(13) 10^{-4}$ \\
\hline & & $3 d$ & $2.8598(5)$ & & $0.0^{a}$ & $5.02(3) 10^{-5}$ \\
\hline & & $\overline{2 p}$ & $3.98928(8)$ & $1.85965(3)$ & $0.172589(8)$ & \\
\hline \multirow[t]{4}{*}{$\overline{\mathrm{F}}$} & $1.310(3)$ & $1 s$ & $9.232(2)$ & $7.037(14)$ & $\#$ & 1.0 \\
\hline & & $2 s$ & $7.0901(19)$ & $2.1520(2)$ & $\#$ & $-1.30(6) 10^{-2}$ \\
\hline & & $2 p$ & $4.4857(4)$ & $2.00946(14)$ & $0.15308(6)$ & $-9.15(19) 10^{-3}$ \\
\hline & & $3 s$ & $3.0007(7)$ & & $0.0^{a}$ & $7.92(15) 10^{-4}$ \\
\hline $\mathrm{Ne}$ & & $3 d$ & $2.74243(12)$ & & $0.0^{a}$ & $-1.102(7) 10^{-4}$ \\
\hline \multirow[t]{6}{*}{$\mathrm{Na}$} & $1.5469(11)$ & $1 s$ & $11.1214(11)$ & $7.807(19)$ & $\#$ & 1.0 \\
\hline & & $2 s$ & $8.6638(18)$ & $2.82430(13)$ & \# & $-8.43(19) 10^{-3}$ \\
\hline & & $2 p$ & $5.7308(3)$ & $2.78883(12)$ & $0.19053(5)$ & $-5.69(17) 10^{-3}$ \\
\hline & & $3 s$ & $3.8585(14)$ & & $0.0^{a}$ & $2.86(12) 10^{-4}$ \\
\hline & & $3 p$ & $3.1693(8)$ & & $0.0^{a}$ & $1.26(8) 10^{-4}$ \\
\hline & & $\frac{1}{3 s}$ & $1.53832(2)$ & $0.7536(16)$ & $0.135076(3)$ & \\
\hline \multirow[t]{5}{*}{$\mathrm{Mg}$} & $1.692(6)$ & $1 s$ & $12.0983(14)$ & $8.12(4)$ & $\#$ & 1.0 \\
\hline & & $2 s$ & 9.3103(19) & $3.2525(3)$ & \# & $1.0^{b}$ \\
\hline & & $2 p$ & $6.3862(5)$ & $3.28864(16)$ & $0.22040(7)$ & $1.0^{b}$ \\
\hline & & $3 s$ & $1.7571(2)$ & $0.96373(13)$ & $0.11295(7)$ & $1.986(10) 10^{-4}$ \\
\hline & & $3 p$ & $3.417(15)$ & $1.2284(2)$ & $0.439(11)$ & $-2.825(15) 10^{-5}$ \\
\hline \multirow[t]{6}{*}{$\overline{\mathrm{Al}}$} & $1.840(8)$ & $1 s$ & $13.084(2)$ & $8.47(9)$ & $\#$ & 1.0 \\
\hline & & $2 s$ & $9.826(8)$ & $3.6344(12)$ & $\#$ & $1.0^{b}$ \\
\hline & & $2 p$ & $7.0532(16)$ & $3.7780(8)$ & $0.2597(3)$ & $1.0^{b}$ \\
\hline & & $3 s$ & $2.190(3)$ & $1.2568(11)$ & $0.1487(6)$ & $7.781(13) 10^{-4}$ \\
\hline & & $3 p$ & $5.58(2)$ & $1.5531(7)$ & $0.1416(7)$ & $-1.004(10) 10^{-4}$ \\
\hline & & $\frac{1}{3 p}$ & $1.916(3)$ & $0.9374(6)$ & $0.1282(5)$ & \\
\hline \multirow[t]{5}{*}{$\overline{\mathrm{Si}}$} & $1.968(3)$ & $1 s$ & $14.0697(7)$ & $8.68(4)$ & $\#$ & 1.0 \\
\hline & & $2 s$ & $10.4077(18)$ & $4.0249(4)$ & $\#$ & $1.0^{b}$ \\
\hline & & $2 p$ & $7.7030(7)$ & $4.2614(3)$ & $0.29444(12)$ & $1.0^{b}$ \\
\hline & & $3 s$ & $2.4437(3)$ & $1.42785(13)$ & $0.12038(3)$ & $7.327(16) 10^{-4}$ \\
\hline & & $3 p$ & $6.110(12)$ & $1.8471(3)$ & $0.19670(14)$ & $-8.961(18) 10^{-5}$ \\
\hline
\end{tabular}

${ }^{a}$ In the case of dynamic correlation, we used single zeta orbitals beyond the $H F$ ones.

${ }^{b}$ For $M g, A l$ and $S i$, we optimized only the $\lambda$ 's related to the $3 s-$ 
TABLE V: AGP+J (three body) wavefunctions

The notations are the same as in Table III The parametrization for the three-body Jastrow factor is also reported.

$B e$

\begin{tabular}{|c|c|c|c|c|c|}
\hline geminal & $Z_{1}$ & $\overline{Z_{2}}$ & $p$ & $\bar{\lambda}$ & \\
\hline $1 s$ & $7.48(8)$ & $3.545(2)$ & $\#$ & 1.0 & \\
\hline $2 p$ & $2.86(5)$ & $1.1064(9)$ & $1.245(9)$ & $-6.27(9) 10^{-4}$ & \\
\hline Jastrow 2 body & $0.7935(19)$ & & & & \\
\hline Jastrow 3 body & $\overline{\bar{Z}}$ & $\overline{\bar{Z}} \overline{Z_{2}}$ & $a_{0}$ & $a_{1}$ & $\overline{\overline{a_{2}}}$ \\
\hline \multicolumn{6}{|l|}{$M g$} \\
\hline geminal & $Z_{1}$ & $Z_{2}$ & $p$ & $\lambda$ & \\
\hline $1 s$ & 12.0 & 0.0 & $\#$ & 1.0 & \\
\hline $2 s$ & $10.784(2)$ & $3.1685(4)$ & $\#$ & $2.40(18) 10^{-1}$ & \\
\hline $2 p$ & $6.6199(4)$ & $3.25296(19)$ & $0.19285(9)$ & $8.56(2) 10^{-1}$ & \\
\hline Jastrow 3 body & $Z_{1}$ & $Z_{2}$ & $a_{0}$ & $a_{1}$ & $a_{2}$ \\
\hline$\psi_{0}(r)^{c}$ & $1.465(6)$ & & $-0.3919(5)$ & $1.3867(9)$ & $-0.891(2)$ \\
\hline $\bar{\psi}_{1}(\mathbf{r})^{b}$ & $13.03(3)$ & $1.074(10)$ & $0.8789(2)$ & $0.29822(5)$ & \\
\hline
\end{tabular}

${ }^{a} \psi_{0}(r)=a_{0}\left(\exp \left(-Z_{1} r^{2}\right)+a_{1} \exp \left(-Z_{2} r^{2}\right)+a_{2}\right)$

${ }^{b} \bar{\psi}_{1}(\mathbf{r})=\mathbf{r} a_{0}\left(\exp \left(-Z_{1} r^{2}\right)+a_{1} \exp \left(-Z_{2} r^{2}\right)\right)$

${ }^{c} \psi_{0}(r)=a_{0}\left(\left(1+a_{1} r^{2}\right) \exp \left(-Z_{1} r^{2}\right)+a_{2}\right)$

\section{Variational Monte Carlo}

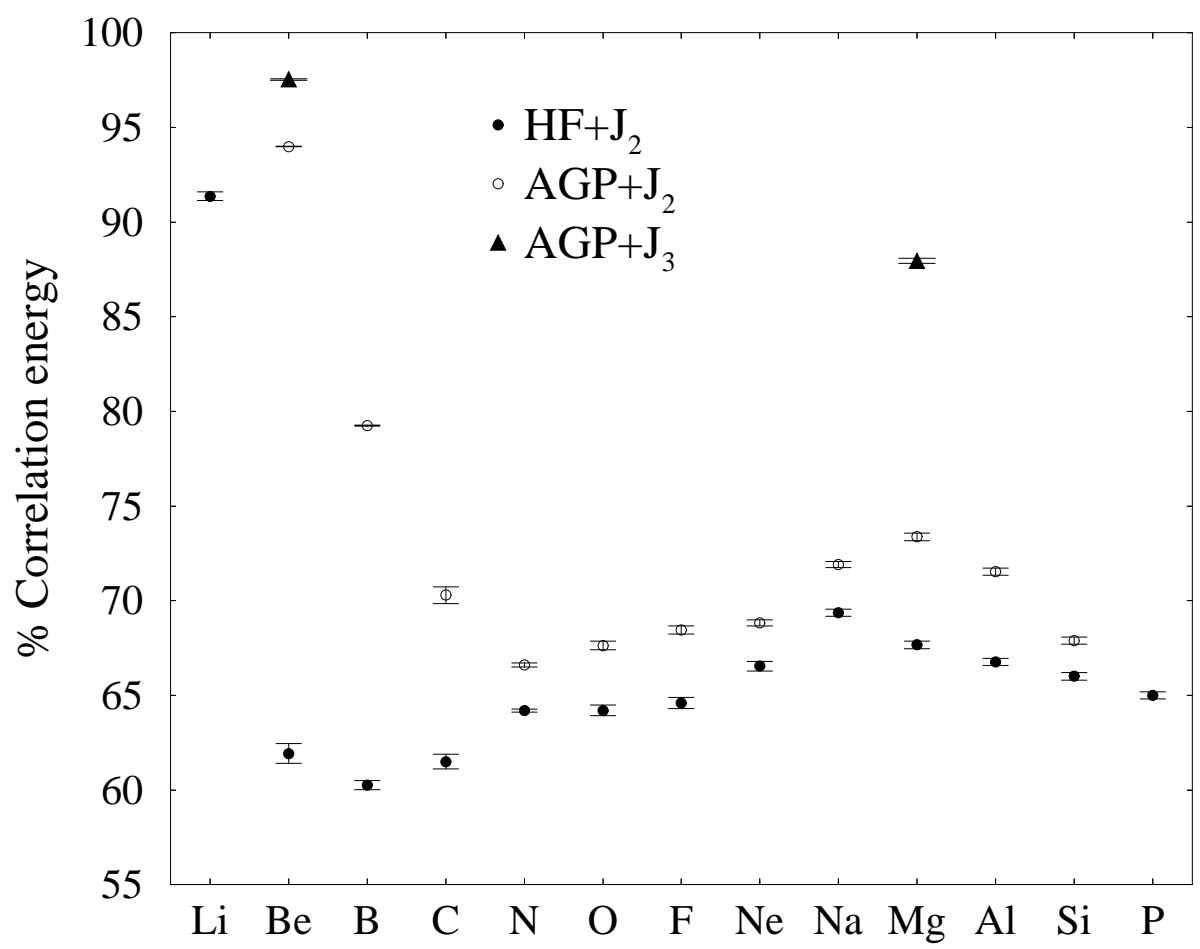

FIG. 1: VMC correlation energies for $H F+J_{2}$ (minimal geminal expansion with a two-body Jastrow factor), $A G P+J_{2}$ (best geminal expansion) and $A G P+J_{3}$ (best geminal with a three-body Jastrow factor) 


\section{Diffusion Monte Carlo}

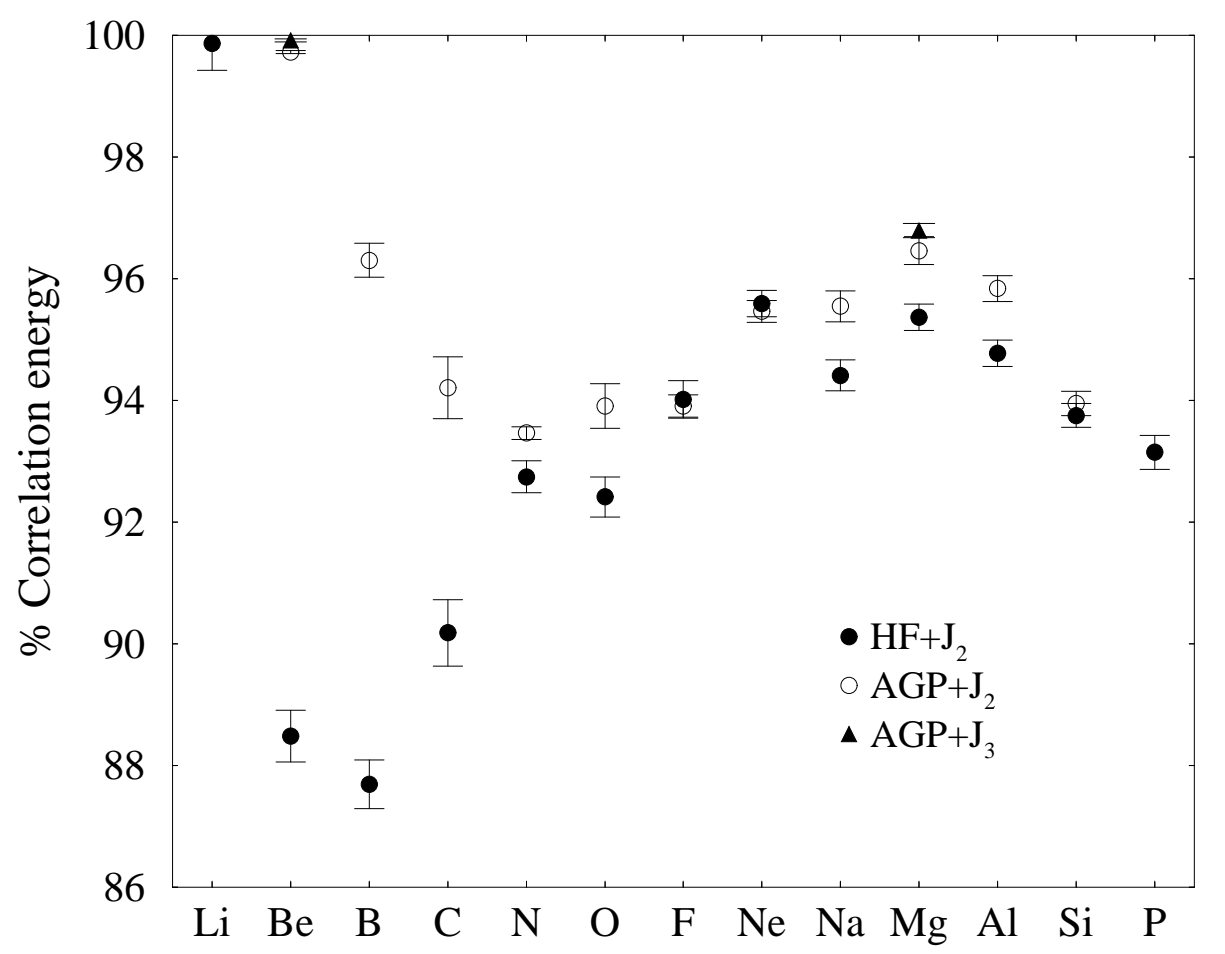

FIG. 2: DMC correlation energies obtained by $H F+J_{2}, A G P+J_{2}$ and $A G P+J_{3}$ wavefunctions 